\title{
CONFORTO TÉRMICO EM COLÔNIAS DE Tetragonisca weyrauchi NO MUNICÍPIO DE RIO BRANCO-ACRE
}

\author{
Francisco Cildomar da Silva Correia ${ }^{1}$ \\ Marcelo Bastos Cordeiro ${ }^{1}$ \\ Yuri Karaccas de Carvalho ${ }^{1}$ \\ Rui Carlos Peruquetti ${ }^{1}$
}

CORREIA, F. C. da S.; CORDEIRO, M. B.; CARVALHO, Y. K. de; PERUQUETTI, R. C. Conforto térmico em colônias de Tetragonisca weyrauchi no município de Rio Branco-Acre. Arq. Ciênc. Vet. Zool. UNIPAR, Umuarama, v. 18, n. 4, p. 237-240, out./dez. 2015.

RESUMO: A espécie Tetragonisca weyrauchi é capaz de manter a temperatura interna do ninho em homeostase, variando entre 29 e $35^{\circ} \mathrm{C}$, embora ainda não se conheçam os mecanismos usados por esse grupo de insetos para manter o controle da temperatura interna da colônia.O controle termorregulatório no interior da colônia pode ser importante para incubação da cria e para sobrevivência da colmeia, em temperaturas extremas. Para o monitoramento das temperaturas utilizou-se Data Logger (modelo HOBO U12 - 012 com exatidão de $\pm 0,35^{\circ} \mathrm{C}$ e sensor para medição interna). A temperatura interna foi obtida com sensor Data Logger, introduzido na região dos favos de cria, por meio de um furo na lateral do ninho; já a temperatura externa foi obtida com Data Logger, mantido a $10 \mathrm{~cm}$ da colmeia. Para verificar a existência do controle termorregulatório da colônia, a temperatura externa foi comparada com a temperatura interna, utilizando-se a correlação de Pearson $\left(\mathrm{R}^{2}=0,514 ; \mathrm{n}=1159\right.$; $\mathrm{p}<0,05)$ para determinar os parâmetros de correlação entre as variáveis.O presente estudo demonstrou que existe controle termorregulatório no interior do ninho de $T$. weyrauchi, que apresentou pouca variação em relação à temperatura externa, ficando em $5,7^{\circ} \mathrm{C}$ entre a temperatura mínima e a máxima, no decorrer da investigação.

PALAVRAS-CHAVE: Abelha sem ferrão. Ninho. Temperatura.

\section{THERMAL COMFORT IN Tetragonisca weyrauchi COLONY THE MUNICIPALITY OF RIO BRANCO-ACRE}

\begin{abstract}
The Tetragonisca weyrauchi species is able to maintain the internal temperature of the nest in homeostasis, ranging between 29 and $35^{\circ} \mathrm{C}$, although it is not known yet the mechanisms used by this group of insects to maintain control of the internal temperature of the colony. The thermoregulatory control within the colony may be important for establishing incubation and hive survival in extreme temperatures. For monitoring temperatures was used Data Logger (model HOBO $\mathrm{U} 12$ - 012 with accuracy of $\pm 0.35^{\circ} \mathrm{C}$ and sensor for internal measurement). The internal temperature was obtained with the sensor (Data Logger) introduced in the region of the combs through a hole beside the nest, also the external temperature was obtained with data logger held $10 \mathrm{~cm}$ from the hive. To check the existence of thermoregulatory control of the colony, the external temperature was compared with the internal temperature using thePearson correlation $\left(\mathrm{R}^{2}=0,514 ; \mathrm{n}=1159 ; \mathrm{p}<0,05\right)$ to determine the parameters of correlation between the variables. This study .demonstrated that there is thermoregulatory control inside the nest $T$. weyrauchi that showed a little change from the outside temperature, remaining at $5.7^{\circ} \mathrm{C}$ between the minimum and the maximum temperature during the investigation.
\end{abstract}

KEYWORDS: Nest. Stingless bees. Temperature.

\section{CONFORT TÉRMICO EN COLONIAS DE Tetragonisca weyrauchi EN EL MUNICIPIO DE RIO BRANCO,} ACRE

RESUMEN: La especie Tetragonisca weyrauchi es capaz de mantener la temperatura interna del nido en homeostasis, que oscilan entre 29 y $35^{\circ} \mathrm{C}$, aunque todavía no conocemos los mecanismos utilizados por ese grupo de insectos para mantener el control de la temperatura interior de la colonia. El control termorregulador dentro de la colonia puede ser importante para eclosión de la cría y para supervivencia de la colmena, en temperaturas extremas. Para el control de las temperaturas se ha utilizado Data Logger (modelo HOBO U12 - 012 con precisión de $\pm 0,35^{\circ} \mathrm{C}$ y sensor para medición interna). La temperatura interna se obtuvo con el sensor Data Logger introducido en la región de los panales de cría, a través de un agujero en la lateral del nido; ya la temperatura externa ha sido obtenida con Data Logger, mantenido a 10cm de distancia de la colmena. Para comprobar la existencia del control termorregulador de la colonia, la temperatura externa ha sido comparada con la temperatura interna, utilizándose la correlación de Pearson $\left(\mathrm{R}^{2}=0,514 ; \mathrm{n}=1159 ; \mathrm{p}<0,05\right)$ para determinar los parámetros de correlación entre las variables. El presente estudio ha demostrado que existe control termorregulador en el interior del nido de $T$. weyrauchi, que mostró poca variación en relación a la temperatura externa, quedando en $5,7^{\circ} \mathrm{C}$ entre la temperatura mínima y la máxima, en el curso de la investigación.

PALABRAS CLAVE: Abejas sin aguijón. Nido. Temperatura.

DOI: https://doi.org/10.25110/arqvet.v18i4.2015.5750

${ }^{1}$ Centro de Ciências Biológicas e da Natureza, Universidade Federal do Acre - PPGESPA, Rod. BR 364, km 4 - Distrito Industrial, Bloco EdilbertoParigot de Souza Filho - $2^{\circ}$ piso. Rio Branco, Acre - Brasil - CEP 69920-900. E-mail: cildomar12@hotmail.com 


\section{Introdução}

Abelhas sem ferrão fazem parte da ordem Hymenoptera, família Apidae e subfamília Apinae. São grandes produtoras de mel, cera e geoprópolis. Além disso, são indispensáveis para o equilíbrio dos ecossistemas, uma vez que são as principais polinizadoras de angiospermas nas regiões tropicais e subtropicais do planeta, sendo responsáveis por cerca de $90 \%$ da polinização de plantas nativas e de várias espécies de plantas cultivadas (KERR et al., 2001).

Por outro lado, as abelhas são dependentes das angiospermas, por esse grupo de plantas garantirem quase todos os recursos que as abelhas necessitam como néctar, pólen, resina, óleo, proteção aos ninhos, dentre outros insumos importantes para sua sobrevivência e para a manutenção da colônia (WITTER et al., 2014).

Existem aproximadamente 30.000 espécies de abelhas descritas (GRISWOLD; PARKER; HANSON, 1995), formando 33 gêneros (SILVEIRA; MELO; ALMEIDA, 2002), que agrupam cerca de 400 espécies pertencentes à tribo Meliponini, as quais são conhecidas como abelhas sem ferrão, em virtude da sua incapacidade de ferroar. Uma delas é a $T$. weyrauchi, espécie popularmente conhecida como jataí. A tribo Meliponini encontra-se entre os principais grupos de abelhas sem ferrão, que estão distribuídas entre os trópicos de Câncer e de Capricórnio, espalhadas entre a África, Oceania e América tropical (SAKAGAMI, 1982). A espécie T. weyrauchi pode ser encontrada no Peru, Bolívia e Brasil (Acre, Rondônia e Mato Grosso) (CAMARGO; PEDRO, 2013).

As abelhas do gênero Tetragonisca são versáteis, em relação ao local de nidificação, comumente, constroem seus ninhos em ocos de árvores localizados, com frequência, próximos ao solo. No entanto, em regiões urbanizadas, utilizam ocos de tijolos e outros orifícios (CORTOPASSI-LAURINO; NOGUEIRA-NETO, 2003). A espécie T. weyrauchi, encontrada no Acre, constrói seus ninhos em forquilhas de galhos de árvores. O ninho é construído inteiramente de cerume, com pequenos furos nas paredes e respiráculo na parte superior, o que permite o controle da ventilação interna e a dissipação de calor. Possui forma cilíndrica com as extremidades superior e inferior estreitadas, apresenta aproximadamente $35 \mathrm{~cm}$ de altura, $60 \mathrm{~cm}$ de circunferência no centro e $30 \mathrm{~cm}$ nas extremidades inferior e superior, sendo coberto por uma película fina e levemente brilhante, na cor castanho-escuro. A entrada do ninho está localizada na sua parte inferior e corresponde a um pequeno tubo de três a quatro centímetros de comprimento e de um a dois centímetros de diâmetro, permitindo a passagem de várias abelhas ao mesmo tempo. Esta entrada é vedada à noite e reaberta pela manhã, podendo continuar fechada em dias frios (CORTOPASSI-LAURINO; NOGUEIRA-NETO, 2003).

O controle termorregulatório no interior da colônia pode ser importante para incubação da cria e sobrevivência da colmeia, em temperaturas extremas (LOLI, 2008; CARVALHO, 2009; DOMINGOS; GONÇALVES, 2014). A termorregulação colonial pode ocorrer por mecanismos comportamentais, tais como: escolha do local de nidificação, construção do invólucro e lamelas, nas regiões dos discos de cria ou por mecanismos fisiológicos, dentre eles: termogênese, por meio da vibração da musculatura, comportamento de voo e pela troca de calor corporal (JONES; OLDROYD, 2007). Alguns meliponicultores costumam desenvolver colmeias especiais com sistemas de termorregulação artificial para manutenção e desenvolvimento dos ninhos, durante o inverno (VOLLET; MENEZEZ; FONSECA, 2010).

A capacidade termorreguladora em colônias de abelhas sem ferrão pode ser atribuída às características estruturais do ninho, como invólucro e batume, que podem apresentar bom isolamento térmico (ZUCCHI; SAKAGAMI, 1972). Os resultados de Campos, Gois e Medeiros (2010), que encontraram registros de variações na termorregulação, em diferentes espécies de abelhas do gênero Melipona, foram confirmados por Roldão (2011), ao afirmar que as abelhas sem ferrão são capazes de manter a temperatura interna do ninho mais ou menos estável, entre 25 e $32{ }^{\circ} \mathrm{C}$, variando de acordo com a espécie, com a colônia e com a exposição ao sol.

A espécie $T$. weyrauchi é capaz de manter a temperatura interna do ninho em homeostase, variando entre 29 e $35^{\circ} \mathrm{C}$, embora ainda não se conheçam os mecanismos usados por esse grupo de abelhas para manter o controle da temperatura interna da colônia.

\section{Material e Métodos}

As observações foram realizadas em uma área rural próximo a Rio Branco, Acre (9॰52'58' S, $67^{\circ} 52^{\prime}$ '8” W), entre 29/07/2015 e 11/08/2015, 24 horas por dia, em um ninho de T.weyrauchi, protegido do sol e da chuva por uma cobertura de palha, sendo que as temperaturas interna e externa foram medidas, simultaneamente, a cada 15 minutos. Esses dados foram coletados e armazenados em computador. Para o monitoramento das temperaturas utilizou-se Data Logger (modelo HOBO U12 - 012, com exatidão de $\pm 0,35^{\circ} \mathrm{C}$ e sensor para medição interna).A temperatura interna foi obtida com sensor, introduzido na região dos discos de cria, por meio de um furo na lateral do ninho; já a temperatura externa foi obtida com Data Logger,mantido a $10 \mathrm{~cm}$ da colmeia.

Para se verificar a existência do controle termorregulatório do ninho, a temperatura externa foi comparada com a temperatura interna,utilizando-se a Correlação Linear de Pearson. As comparações foram feitas considerando-se as medidas das temperaturas tomadas a cada 15 minutos, com uma probabilidade de erro de 5\% (nível de significância) e um nível de confiança de $95 \%$.

\section{Resultados e Discussão}

Observou-se que a temperatura externa apresentou variações ao longo dos dias no presente estudo. No geral, a temperatura aumentou gradativamente entre 06:00 e 15:00 horas, com picos de temperatura às 14:30 minutos, diminuindo a partir das 16:00 horas (Figura 2). A temperatura máxima registrada foi de $39,5{ }^{\circ} \mathrm{C}$, às $14: 30$ minutos, no dia $04 / 08 / 2015$ e a mínima foi de $18,2{ }^{\circ} \mathrm{C}$, às $05: 45$ minutos, no dia $31 / 07 / 2015$, sendo que a temperatura interna do ninho de T.weyrauchise manteve entre 29 e $35^{\circ} \mathrm{C}$, sofrendo pequena variação de $5,7^{\circ} \mathrm{C}$, no decorrer das observações (Figuras 1 e 3).

Em estudos realizados por Cortopassi-Laurino e Nogueira-Neto (2003), na cidade de Xapuri-Acre, verificou-se que a temperatura interna do ninho de T. weyrauchi acom- 
panha as variações da temperatura externa, o que contraria as avaliações realizadas na cidade de Rio Branco-Acre, em uma colônia dessa mesma espécie, que se mostrou eficiente no controle temorregulatório interno, mantendo-se entre 29 e $35^{\circ} \mathrm{C}$. A correlação entre a temperatura interna da colônia e a do ambiente (correlação de Pearson, $\mathrm{R}^{2}=0,514$; $\mathrm{n}=1159$; $\mathrm{p}<0,05)$ apresentou variações muito pequenas ao longo do dia, se comparada à da temperatura externa (Figura 2). Desse modo, a variação da temperatura interna da colônia de $T$. weyrauchi é pouco influenciada pela variação da temperatura externa (temperatura ambiente), não podendo explicar a variação da temperatura nos discos de cria como sendo função apenas da variação da temperatura ambiente, como sugerido por Cortopassi-Laurino e Nogueira-Neto (2003).

Durante os 14 dias de estudos, a temperatura externa variou entre 18 a $39^{\circ} \mathrm{C}$, devido aos ventos frios de origem polar, fenômeno conhecido como friagem, que ocorre entre os meses de maio a agosto, provocando quedas bruscas na temperatura durante a noite e início da manhã, apresentando diferenças de até $21^{\circ} \mathrm{C}$ ao longo do dia, como pode ser observado na Figura 1.

Figura 1: Relação entre as temperaturas interna e externa nos dias de coleta.

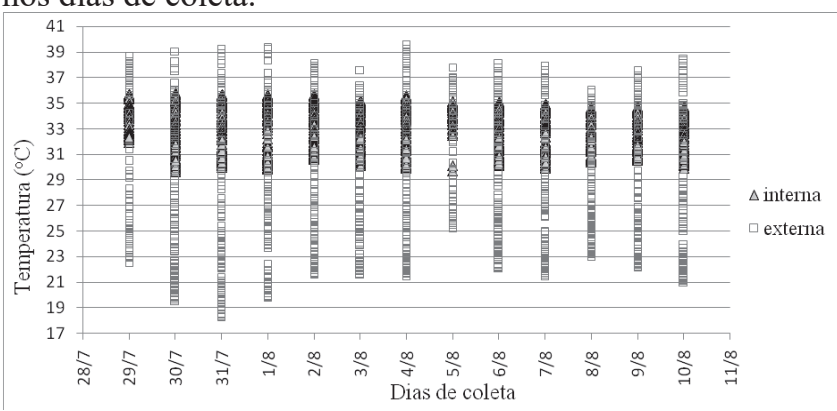

A média da variação de temperatura dentro do ninho foi de $5,7{ }^{\circ} \mathrm{C}$, sendo que a menor temperatura registrada foi de $29,9^{\circ} \mathrm{C}$, às $05: 45$ minutos, no dia $31 / 07 / 2015$, e a maior foi de $35,6^{\circ} \mathrm{C}$, às $11: 30$ minutos, no dia $04 / 08 / 2015$.

Figura 2: Relação entre o equilíbrio termorregulatório do interior da colmeia e a temperatura ambiente.

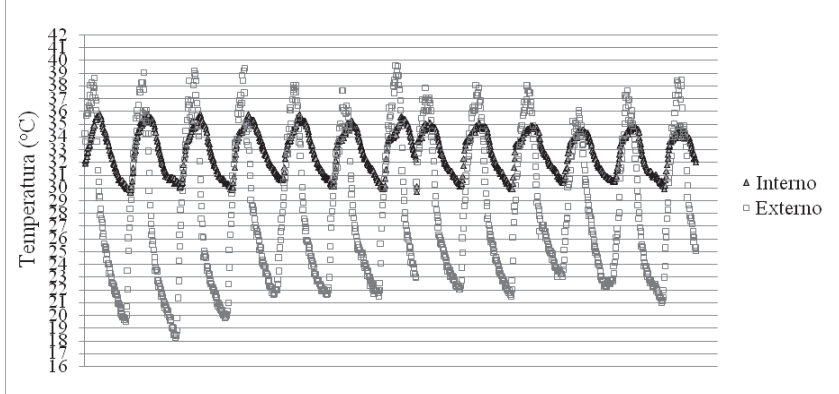

A Figura 3 mostra a correlação entre as temperaturas interna e externa (correlação de Pearson, $\mathrm{R}^{2}=0,514$; $\mathrm{n}=1159 ; \mathrm{p}<0,05)$,demonstrando que a temperatura interna da colmeia de T.weyrauchi é pouco influenciada pela temperatura externa (temperatura ambiente), comprovando que a temperatura nos discos de cria não pode ser explicada pela variação da temperatura ambiente.
Figura 3: Análise do coeficiente de correlação e coeficiente de determinação.

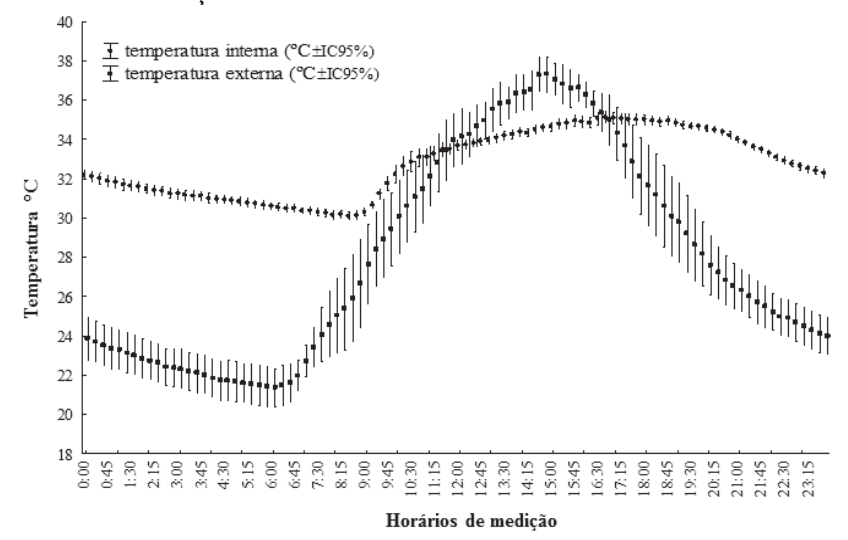

Os dados sugerem a existência de ação termorregulatória ativa em colônias de $T$. weyrauchi, visto que essa espécie de abelha consegue manter a temperatura interna do ninho entre $29^{\circ} \mathrm{C}$ e $35^{\circ} \mathrm{C}$ o que, acredita-se, estar dentro da sua zona de conforto térmico.

\section{Conclusão}

O estudo realizado em uma colônia de T. weyrauchi demonstrou que existe controle termorregulatório no interior do ninho (região dos discos de cria), que apresentou pouca variação em relação às oscilações da temperatura externa, ficando em $5,7^{\circ} \mathrm{C}$ entre a temperatura mínima e a máxima observada no decorrer da investigação, o que abre precedentes para futuras pesquisas em busca de se conhecer os mecanismos utilizados por esse grupo de abelhas na termorregulação de seus ninhos.

\section{Referências}

CAMARGO, J. M. F.; PEDRO, S. R. M. Meliponini Lepeletier, 1836. In: MOURE, J. S.; URBAN, D.; MELO, G. A. R. Catalogue of Bees(Hymenoptera, Apoidea) in the Neotropical Region, 2013. Disponível em: <>. Acesso em: 23 out. 2015 .

CAMPOS, F. S.; GOIS, G. C.; CARNEIRO, G. G. Termorregulação colonial em abelhas sem ferrão. PUBVET, Londrina, v. 4, n. 24, ed. 129, 2010.

CARVALHO, M. D. F. Temperatura da superfície corpórea e perda de calor por convecção em abelhas (Apis mellifera) em uma região semi-árida. 2009. $47 \mathrm{f}$. Dissertação (Mestrado em Ciência Animal) - Universidade Federal Rural do Semi-árido, Mossoró, 2009.

CORTOPASSI-LAURINO, M.; NOGUEIRA-NETO, P. Notas Sobre a Bionomia de Tetragonisca weyrauchi Scharz, 1943 (Apidae, Meliponini). Acta Amazônica, Manaus, v. 33, n. 4, p. 643-650, 2003.

DOMINGOS, H. G. T.; GONÇALVES, L. S.

Termorregulação de abelhas com ênfase em Apis mellifera. Acta Veterinaria Brasilica, Mossoró, v. 8, n. 3, p. 150-154, 2014. 
GRISWOLD, T.; PARKER, F. D.; HANSON, P. E. The bees (Apidae). In: HANSON, P. E.; GAULD, I. D. The Hymenoptera of Costa Rica. Oxford: Oxford University Press, 1995.893 p.

JONES, J. C.; OLDOYD, B. P. Nest thermorregulation in social insects. Advances in Insect Physiogy, v. 33, p. 153 191, 2007.

KERR, W. E. et al. Aspectos pouco mencionados da biodiversidade amazônica. In: ABREU, A. R. et al. Biodiversidade, pesquisa e desenvolvimento na Amazônia: parcerias estratégicas. Brasília: Ministério da Ciência e Tecnologia, 2001. p. 20-41.

LOLI, D. Termorregulação colonial e energética individual em abelhas sem ferrão Melipona quadrifasciata Lepeletier (Hymenoptera, Apidae, Meliponini). 2008. 228 f. Tese (Doutorado em Ciências) - Departamento de Fisiologia, Instituto de Biociência da Universidade de São Paulo, São Paulo, 2008.

ROLDÃO, Y. S. Termorregulação colonial e a influência da temperatura no desenvolvimento da cria em abelhas sem ferrão, Melipona scutellaris (Hymenoptera, Apidae, Meliponini). 2011. 107 f. Dissertação (Mestrado em Entomologia) - FFCLRP-USP, Universidade de São Paulo, Ribeirão Preto, 2011.

SAKAGAMI, S. F. Stingless bees. In: HERMANN, H. R. Social insects, New York: Academic Press, p. 361-423, 1982.

SILVEIRA, F. A.; MELO, G. A. R.; ALMEIDA, E. A. B. Abelhas brasileiras: sistemática e identificação. Belo Horizonte: Composição e Arte, 2002. v. 1.

VOLLET, N. D.; MENEZEZ, C.; FONSECA, V. L. I. Aquecimento de colmeias de abelhas sem ferrão: Vale a pena? Mensagem Doce, v. 1, p. 16-20, 2010.

WITTER, S. et al. As abelhas e a agricultura. Porto Alegre: EDIPUCRS, 2014. 143 p.

ZUCCHI, R.; SAKAGAMI, S. F. 1972. Capacidade termoreguladora em Trigona spinipes e em algumas outras espécies de abelhas sem ferrão (Hymenoptera: Apidae: Meliponinae), p. 301- 309. In: CRUZ-LANDIM, C. et al. Livro em homenagem a Warwik Estevan Kerr. Rio Claro: UNESP, 1972. 315 p. 